\title{
Solutions to the Allen Cahn Equation and Minimal Surfaces
}

Manuel del Pino \& Juncheng Wei

\section{Milan Journal of Mathematics}

Issued by the Seminario Matematico e Fisico di Milano

ISSN 1424-9286

Volume 79

Number 1

Milan J. Math. (2011) 79:39-65

DOI 10.1007/s00032-011-0155-6

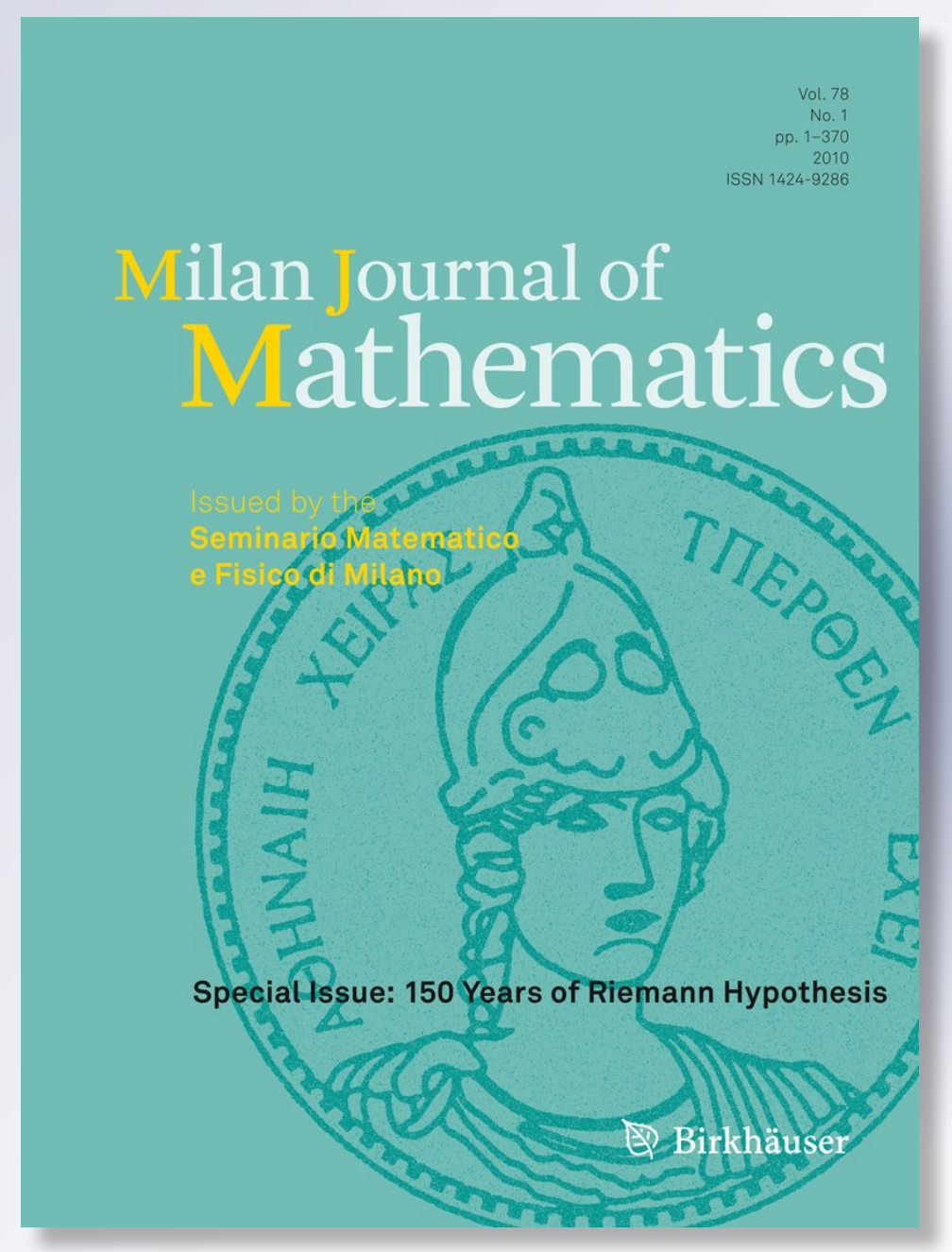

黛 Springer 
Your article is protected by copyright and all rights are held exclusively by Springer Basel AG. This e-offprint is for personal use only and shall not be self-archived in electronic repositories. If you wish to self-archive your work, please use the accepted author's version for posting to your own website or your institution's repository. You may further deposit the accepted author's version on a funder's repository at a funder's request, provided it is not made publicly available until 12 months after publication. 


\title{
Solutions to the Allen Cahn Equation and Minimal Surfaces
}

\author{
Manuel del Pino and Juncheng Wei
}

\begin{abstract}
We discuss and outline proofs of some recent results on application of singular perturbation techniques for solutions in entire space of the Allen-Cahn equation $\Delta u+u-u^{3}=0$. In particular, we consider a minimal surface $\Gamma$ in $\mathbb{R}^{9}$ which is the graph of a nonlinear entire function $x_{9}=F\left(x_{1}, \ldots, x_{8}\right)$, found by Bombieri, De Giorgi and Giusti, the BDG surface. We sketch a construction of a solution to the Allen Cahn equation in $\mathbb{R}^{9}$ which is monotone in the $x_{9}$ direction whose zero level set lies close to a large dilation of $\Gamma$, recently obtained by $\mathrm{M}$. Kowalczyk and the authors. This answers a long standing question by De Giorgi in large dimensions (1978), whether a bounded solution should have planar level sets. We sketch two more applications of the BDG surface to related questions, respectively in overdetermined problems and in eternal solutions to the flow by mean curvature for graphs.
\end{abstract}

Keywords. Minimal surfaces, Infinite dimensional Lyapunov-Schmidt reduction, Jacobi operator.

\section{Introduction}

The Allen-Cahn equation in $\mathbb{R}^{N}$ is the semilinear elliptic problem

$$
\Delta u+u-u^{3}=0 \text { in } \mathbb{R}^{N} .
$$

Originally formulated in the description of bi-phase separation in fluids and ordering in binary alloys [1], Equation (1.1) has received extensive mathematical study. It is a prototype for the modeling of phase transition phenomena in a variety of contexts.

Introducing a small positive parameter $\varepsilon$ and writing $v(x):=u\left(\varepsilon^{-1} x\right)$, we get the scaled version of (1.1),

$$
\varepsilon^{2} \Delta v+v-v^{3}=0 \quad \text { in } \mathbb{R}^{N} .
$$

This work has been supported by FONDECYT grant 1110181, Anillo ACT 125 and an earmarked grant from Hong Kong. 
On every bounded domain $\Omega \subset \mathbb{R}^{N},(1.1)$ is the Euler-Lagrange equation for the action functional

$$
J_{\varepsilon}(v)=\int_{\Omega} \frac{\varepsilon}{2}|\nabla v|^{2}+\frac{1}{4 \varepsilon}\left(1-v^{2}\right)^{2} .
$$

We observe that the constant functions $v= \pm 1$ minimize $J_{\varepsilon}$. They are idealized as two stable phases of a material in $\Omega$. It is of interest to analyze configurations in which the two phases coexist. These states are represented by stationary points of $J_{\varepsilon}$, or solutions $v_{\varepsilon}$ of Equation (1.2), that take values close to +1 in a subregion of $\Omega$ of and -1 in its complement. Modica and Mortola [29] and Modica [28], established that a family of local minimizers $v_{\varepsilon}$ of $J_{\varepsilon}$ for which

$$
\sup _{\varepsilon>0} J_{\varepsilon}\left(v_{\varepsilon}\right)<+\infty
$$

must satisfy as $\varepsilon \rightarrow 0$, after passing to a subsequence,

$$
v_{\varepsilon} \rightarrow \chi_{\Lambda}-\chi_{\Omega \backslash \Lambda} \quad \text { in } L_{l o c}^{1}(\Omega) .
$$

Here $\Lambda$ is an open subset of $\Omega$ with $\Gamma=\partial \Lambda \cap \Omega$ having minimal perimeter, being therefore a (generalized) minimal surface. Moreover,

$$
J_{\varepsilon}\left(v_{\varepsilon}\right) \rightarrow \frac{2}{3} \sqrt{2} \mathcal{H}^{n-1}(\Gamma) .
$$

$\Gamma$ is then intuitively a surface close to the nodal set of $v_{\varepsilon}$ (or more generally, for a given $\lambda \in(-1,1)$, any level $\left[v_{\varepsilon}=\lambda\right]$ for small $\left.\varepsilon\right)$. Scaling back into equation (1.1), it is then plausible that a certain connection between the level sets of the initial $u$ and the minimal surface $\varepsilon^{-1} \Gamma$ actually takes place, at least when $u$ corresponds to a local minimizer of the energy on each given compact set.

What condition guarantees the locally minimizing (or stability) character of $u$ ? For a solution $u$ of (1.1), this is implied by the fact that the linearized operator $\Delta+\left(1-3 u^{2}\right)$ is positive in the sense of maximum principle. Since the directional derivatives $e \cdot \nabla u$ lie in the kernel of this operator, the assumption that the solution is monotone in some direction, say $\partial_{x_{N}} u>0$ is sufficient for this. De Giorgi's conjecture for the Allen Cahn equation is a statement partly motivated by the above facts, which we state below.

For $n=1$ the function

$$
w(t):=\tanh \left(\frac{t}{\sqrt{2}}\right)
$$

connects monotonically the stable values -1 and +1 and solves (1.1):

$$
w^{\prime \prime}+w-w^{3}=0, \quad w( \pm \infty)= \pm 1, \quad w^{\prime}>0 .
$$

This solution generates a class of solutions to $(\mathrm{AC})$ in the following manner: For any $p, \nu \in \mathbb{R}^{N},|\nu|=1$, the functions

$$
u(x):=w(z), \quad z=(x-p) \cdot \nu
$$

solve equation (1.1). Here $z$ represents the normal coordinate to the hyperplane through $p$, with unit normal $\nu$. A question is whether or not there exist solutions 
connecting the values -1 and 1 along some direction, which are different from these trivial ones.

De Giorgi [15] raised the following conjecture in 1978.

De Giorgi's conjecture: Let $u$ be a bounded solution of equation

$$
\Delta u+u-u^{3}=0 \quad \text { in } \mathbb{R}^{N},
$$

which is monotone in one direction, say $\partial_{x_{N}} u>0$. Then, at least when $N \leq 8$, there exist $p, \nu$ such that

$$
u(x)=w((x-p) \cdot \nu) .
$$

This statement is equivalent to:

At least when $N \leq 8$, all level sets of $u,[u=\lambda]$ must be hyperplanes.

De Giorgi's conjecture is actually a parallel to Bernstein's Problem for minimal surfaces which are entire graphs.

An entire minimal graph in $\mathbb{R}^{N}$ is a surface of the form

$$
\Gamma=\left\{\left(x^{\prime}, F\left(x^{\prime}\right)\right) \in \mathbb{R}^{N-1} \times \mathbb{R} / x^{\prime} \in \mathbb{R}^{N-1}\right\}
$$

where $F$ solves the minimal surface equation in entire space

$$
\nabla \cdot\left(\frac{\nabla F}{\sqrt{1+|\nabla F|^{2}}}\right)=0 \text { in } \mathbb{R}^{N-1} .
$$

Bernstein's problem (by Fleming, 1962): Is it true that all entire minimal graphs are hyperplanes, namely any entire solution of (1.6) must be a linear affine function?

This claim turns out to be True for $N \leq 8$ :

- Bernstein [8] (1917), Fleming [24] (1962) $N=3$.

- De Giorgi [14] (1965) $N=4$.

- Almgren [2] (1966), $N=5$.

- Simons [35](1968), $N=6,7,8$.

It is False for $N \geq 9$ : Bombieri-De Giorgi-Giusti [9] (1969) found a counterexample. De Giorgi's Conjecture: $u$ bounded solution of (1.1), $\partial_{x_{N}} u>0$, then level sets $[u=\lambda]$ are hyperplanes.

- True for $N=2$. Ghoussoub and Gui [25] (1998).

- True for $N=3$. Ambrosio and Cabré [1] (1999).

- True for $4 \leq N \leq 8$. Savin [33] (2009), thesis (2003), if in addition

$$
\lim _{x_{N} \rightarrow \pm \infty} u\left(x^{\prime}, x_{N}\right)= \pm 1 \quad \text { for all } \quad x^{\prime} \in \mathbb{R}^{N-1} \text {. }
$$

A counterexample to De Giorgi's conjecture in dimension $N \geq 9$ was believed to exist for a long time. Partial progress in this direction was made by Jerison and 
Monneau [27] and by Cabré and Terra [10]. See also the survey article by Farina and Valdinoci [22].

\subsection{The Bombieri-De Giorgi-Giusti minimal graph}

The negative example for Berntein's problem in [9] corresponds to an ingenious, and fairly explicit construction by super and sub-solutions of a non-trivial solution to (1.6) when $N=9$ :

$$
H(F):=\nabla \cdot\left(\frac{\nabla F}{\sqrt{1+|\nabla F|^{2}}}\right)=0 \text { in } \mathbb{R}^{8} .
$$

The solution in [9] has the form

$$
F: \mathbb{R}^{4} \times \mathbb{R}^{4} \rightarrow \mathbb{R}, \quad(\mathbf{u}, \mathbf{v}) \mapsto F(|\mathbf{u}|,|\mathbf{v}|) .
$$

In addition, $F(|\mathbf{u}|,|\mathbf{v}|)>0$ for $|\mathbf{v}|>|\mathbf{u}|$ and

$$
F(|\mathbf{u}|,|\mathbf{v}|)=-F(|\mathbf{v}|,|\mathbf{u}|) \text {. }
$$

Let us introduce polar coordinates:

$$
|\mathbf{u}|=r \cos \theta,|\mathbf{v}|=r \sin \theta, \quad \theta \in\left(0, \frac{\pi}{2}\right) .
$$

Computing the mean curvature operator $H(F)$ in (1.7) at $F=F(r, \theta)$ we get

$$
\begin{aligned}
H[F]= & \frac{1}{r^{7} \sin ^{3} 2 \theta} \partial_{r}\left(\frac{F_{r} r^{7} \sin ^{3} 2 \theta}{\sqrt{1+F_{r}^{2}+r^{-2} F_{\theta}^{2}}}\right) \\
& +\frac{1}{r^{7} \sin ^{3} 2 \theta} \partial_{\theta}\left(\frac{F_{\theta} r^{5} \sin ^{3} 2 \theta}{\sqrt{1+F_{r}^{2}+r^{-2} F_{\theta}^{2}}}\right) .
\end{aligned}
$$

Let us look for an approximate solution by separation of variables $F_{0}(r, \theta)=r^{3} g(\theta)$. We compute

$$
\begin{aligned}
H\left[F_{0}\right]= & \frac{1}{r^{7} \sin ^{3} 2 \theta} \partial_{r}\left(\frac{3 r^{7} g \sin ^{3} 2 \theta}{\sqrt{r^{-4}+9 g^{2}+g^{\prime 2}}}\right) \\
& +\frac{1}{r \sin ^{3} 2 \theta} \partial_{\theta}\left(\frac{g^{\prime} \sin ^{3} 2 \theta}{\sqrt{r^{-4}+9 g^{2}+g^{\prime 2}}}\right) .
\end{aligned}
$$

As $r \rightarrow \infty$ we impose the equation $H\left(F_{0}\right)=0$ satisfied. This becomes the ODE

$$
\begin{gathered}
\frac{21 g \sin ^{3} 2 \theta}{\sqrt{9 g^{2}+g^{\prime 2}}+}\left(\frac{g^{\prime} \sin ^{3} 2 \theta}{\sqrt{9 g^{2}+g^{\prime 2}}}\right)^{\prime}=0 \text { in }\left(\frac{\pi}{4}, \frac{\pi}{2}\right), \\
g\left(\frac{\pi}{4}\right)=0=g^{\prime}\left(\frac{\pi}{2}\right) .
\end{gathered}
$$

This problem has a solution $g$ positive in $\left(\frac{\pi}{4}, \frac{\pi}{2}\right)$, see [16].

We check directly that 
- $F_{0}(r, \theta)=r^{3} g(\theta)$ is a subsolution of the minimal surface equation $H(F)=0$ : $H\left(F_{0}\right) \geq 0$

- $F_{0}(r, \theta)$ accurate approximation to a solution of the minimal surface equation:

$$
H\left(F_{0}\right)=O\left(r^{-5}\right) \quad \text { as } r \rightarrow+\infty .
$$

The supersolution of Bombieri, De Giorgi and Giusti can be refined to yield that $F_{0}$ gives the precise asymptotic behavior of $F$. The following was established in $[16]$.

For $\theta \in\left(\frac{\pi}{4}, \frac{\pi}{2}\right)$ we have, for $0<\sigma<1$ and all large $r$,

$$
F_{0}(r, \theta) \leq F(r, \theta) \leq F_{0}(r, \theta)+A r^{-\sigma} \text { as } r \rightarrow+\infty
$$

The following result, by M. Kowalczyk and the authors "disproves" De Giorgi's conjecture in dimension 9 (and hence in any dimension higher).

Theorem $1([16,17])$. Let $\Gamma$ be a BDG minimal graph in $\mathbb{R}^{9}$ and $\Gamma_{\varepsilon}:=\varepsilon^{-1} \Gamma$. Then for all small $\varepsilon>0$, there exists a bounded solution $u_{\varepsilon}$ of $(A C)$, monotone in the $x_{9}$-direction, with

$$
\begin{gathered}
u_{\varepsilon}(x)=w(\zeta)+O(\varepsilon), \quad x=y+\zeta \nu(\varepsilon y), \quad y \in \Gamma_{\varepsilon},|\zeta|<\frac{\delta}{\varepsilon} \\
\lim _{x_{9} \rightarrow \pm \infty} u\left(x^{\prime}, x_{9}\right)= \pm 1 \quad \text { for all } x^{\prime} \in \mathbb{R}^{8} .
\end{gathered}
$$

We shall devote the main part of the remaining of this paper to describing the proof of the above result. We will introduce in certain detail some necessary background and related notation concerning minimal hypersurfaces in $\mathbb{R}^{n}$ and then carry out the proof omitting the proof of some intermediate steps which are contained in $[16]$.

In the last part of the paper we will state some closely related results for questions related to two well-known problems: construction of solutions to overdetermined semilinear equations (a joint work with F. Pacard), and solutions to the mean curvature flow for entire graphs (with P. Daskalopoulos and M. Kowalczyk).

\section{Preliminaries}

\subsection{Preliminaries I: The Laplacian near a hypersurface}

Let $\Gamma$ be a hypersurface embedded in $\mathbb{R}^{n+1}$ and let $\nu$ designate a choice of unit normal. Local coordinates near $\Gamma$ :

$$
x=y+z \nu(y), \quad y \in \Gamma,|z|<\delta .
$$

We will establish the following formula:

$$
\Delta_{x}=\partial_{z z}+\Delta_{\Gamma^{z}}-H_{\Gamma_{z}}(y) \partial_{z}
$$


Here

$$
\Gamma^{z}:=\{y+z \nu(y) / y \in \Gamma\}
$$

$\Delta_{\Gamma^{z}}$ is the Laplace-Beltrami operator on $\Gamma^{z}$ and $H_{\Gamma^{z}}(y)$ its mean curvature. Let $k_{1}, \ldots, k_{n}$ be the principal curvatures of $\Gamma$. Then, as we shall see

$$
\begin{gathered}
H_{\Gamma^{z}}=\sum_{i=1}^{n} \frac{k_{i}}{1-z k_{i}} \\
x=y+z \nu(y), \quad y \in \Gamma, \quad|z|<\delta .
\end{gathered}
$$

Assume that an open region of $\Gamma$ can be described by means of the parametrization

$$
\mathrm{y} \in \omega \subset \mathbb{R}^{n} \mapsto y=y(\mathrm{y}) \in \mathbb{R}^{n+1}
$$

which induce local coordinates

$$
x=y(\mathrm{y})+z \nu(\mathrm{y}),
$$

To compute the Laplacian, we describe the Dirichlet integral (with no region of integration specified)

$$
I=\int\left|D_{x} \varphi(x)\right|^{2} d x
$$

For a function $f(x)$ we write $\tilde{f}(\mathrm{y}, z)=f(x(\mathrm{y}, z))$. Let us set

$$
\mathrm{Y}_{i}=\partial_{i} y, \quad \mathrm{~B}_{i}=\partial_{i} \nu
$$

where $\partial_{i}=\partial_{\mathrm{y}_{i}}$ and write

$$
\mathrm{Y}=D y=\left[\mathrm{Y}_{1} \cdots Y_{n}\right], \quad \mathrm{B}=D \nu=\left[\mathrm{B}_{1} \cdots B_{n}\right] .
$$

Then

$$
\mathrm{X}=D x(\mathrm{y}, z)=\left[\mathrm{Y}_{1}+z \mathrm{~B}_{1} \cdots Y_{n}+z \mathrm{~B}_{n} \nu\right] .
$$

Let us observe that

$$
\mathrm{X}^{T} \mathrm{X}=\left[\begin{array}{cc}
{[\mathrm{Y}+z \mathrm{~B}]^{T}[\mathrm{Y}+z \mathrm{~B}]} & 0 \\
0 & 1
\end{array}\right] .
$$

Then

Denote

$$
\int|D \varphi|^{2} d x=\int(D \tilde{\varphi})^{T}\left(\mathrm{X}^{T} \mathrm{X}\right)^{-1}(D \tilde{\varphi}) \sqrt{\operatorname{det}\left(\mathrm{X}^{T} \mathrm{X}\right)} d \mathrm{y} d z
$$

$$
g(0):=Y^{T} Y, \quad g(z)=[\mathrm{Y}+z \mathrm{~B}]^{T}[\mathrm{Y}+z \mathrm{~B}]
$$

whose coefficients are those of the metric respectively on $\Gamma$ and $\Gamma^{z}$. We shall also denote, as customary $g^{i j}(z)$, the coefficients of the matrix $g(z)^{-1}$. Then

$$
\int|D \varphi|^{2} d x=\int\left(g^{i j}(z) \partial_{i} \tilde{\varphi} \partial_{j} \tilde{\varphi}+\left|\partial_{z} \tilde{\varphi}\right|^{2}\right) \sqrt{\operatorname{det} g(z)} d \mathrm{y} d z .
$$

Let us analyze further the matrix

$$
g(z)=[\mathrm{Y}+z \mathrm{~B}]^{T}[\mathrm{Y}+z \mathrm{~B}]=\mathrm{Y}^{T} \mathrm{Y}+z\left(\mathrm{~B}^{T} \mathrm{Y}+\mathrm{Y}^{T} B\right)+z^{2} \mathrm{~B}^{T} \mathrm{~B} .
$$


Differentiating the equalities $\mathrm{Y}_{j} \cdot \nu=0=\mathrm{Y}_{i} \cdot \nu$ we find

$$
\mathrm{B}^{T} \mathrm{Y}=\mathrm{Y}^{T} B \text {. }
$$

Also,

$$
\mathrm{Y}\left[\mathrm{Y}^{T} \mathrm{Y}\right]^{-1} \mathrm{Y}^{T} \mathrm{~B}=\mathrm{B}
$$

since the columns of $\mathrm{B}$ are in the space spanned by the columns of $\mathrm{Y}$. Using these two facts we easily get

$$
g(z)=[\mathrm{Y}+z \mathrm{~B}]^{T}[\mathrm{Y}+z \mathrm{~B}]=g(0)[I-z A]^{2}
$$

where $A$ is the (symmetric) matrix

$$
A=-\left[\mathrm{Y}^{T} \mathrm{Y}\right]^{-1} \mathrm{Y}^{T} \mathrm{~B} .
$$

The shape operator is the linear map $\mathcal{L}$ of the tangent space $T_{y} \Gamma$ given by

$$
e \in T_{y} \Gamma \mapsto \mathcal{L}[e]=-\nabla_{e} \nu(y) \in T_{y} \Gamma,
$$

where $\nabla_{e} \nu(y)$ is the directional derivative at $y$ of the normal vector field $\nu$ along $e$. The matrix of $\mathcal{L}$ in the basis $\mathrm{Y}_{1}, \ldots, \mathrm{Y}_{n}$ is $A$ with

$$
\mathcal{L}\left[\mathrm{Y}_{j}\right]=-\mathrm{B}_{j}=\mathrm{Y} A_{\cdot j} .
$$

Hence we have precisely

$$
A=-\left[\mathrm{Y}^{T} \mathrm{Y}\right]^{-1} \mathrm{Y}^{T} \mathrm{~B}
$$

This matrix also represents the second fundamental form $A_{\Gamma}$ in the basis $\mathrm{Y}_{1}, \ldots, \mathrm{Y}_{n}$, which is the symmetric bilinear map of $T_{y} \Gamma$ defined as

$$
A_{\Gamma}\left[e_{1}, e_{2}\right]=\mathcal{L}\left[e_{1}\right] \cdot e_{2} \text {. }
$$

The eigenvalues of the matrix $A$ (or just $A_{\Gamma}$ ) do not depend on the particular parametrization chosen. They are by definition the principal curvatures of $\Gamma$ at $y$, $k_{1}(y), \ldots, k_{n}(y)$. Since

$$
g(z)=g(0)\left(I-z A_{\Gamma}\right)^{2}
$$

we get

$$
\sqrt{\operatorname{det} g(z)}=\sqrt{\operatorname{det} g(0)} \operatorname{det}\left(I-z A_{\Gamma}\right)=\sqrt{\operatorname{det} g(0)} \prod_{i=1}^{n}\left(1-k_{i} z\right) .
$$

By taking first variation of the Dirichlet integral we find

$$
\begin{aligned}
\int \nabla \varphi \cdot \nabla \psi d x= & \int g^{i j}(z) \partial_{i} \tilde{\varphi} \partial_{j} \tilde{\psi} \sqrt{\operatorname{det} g(z)} d \mathrm{y} d z \\
& +\int \partial_{z} \tilde{\varphi} \partial_{z} \tilde{\psi} \sqrt{\operatorname{det} g(z)} d \mathrm{y} d z
\end{aligned}
$$

So by integration by parts we get

$$
\begin{aligned}
\int \Delta \varphi \psi d x= & \int \frac{1}{\sqrt{\operatorname{det} g(z)}} \partial_{j}\left(\sqrt{\operatorname{det} g(z)} g^{i j}(z) \partial_{i} \tilde{\varphi}\right) \tilde{\psi} \sqrt{\operatorname{det} g(z)} d \mathrm{y} d z \\
& +\int \frac{1}{\sqrt{\operatorname{det} g(z)}} \partial_{z}\left(\sqrt{\operatorname{det} g(z)} \partial_{z} \tilde{\varphi}\right) \tilde{\psi} \sqrt{\operatorname{det} g(z)} d \mathrm{y} d z
\end{aligned}
$$


Now, the Laplace-Beltrami operator of the manifold $\Gamma_{z}$ in coordinates $y$ is precisely

$$
\Delta_{\Gamma^{z}}=\frac{1}{\sqrt{\operatorname{det} g(z)}} \partial_{j}\left(\sqrt{\operatorname{det} g(z)} g^{i j}(z) \partial_{i}\right)
$$

while

$$
\frac{1}{\sqrt{\operatorname{det} g(z)}} \partial_{z}\left(\sqrt{\operatorname{det} g(z)} \partial_{z}\right)=\partial_{z z}+\partial_{z} \log \operatorname{det}\left(I-z A_{\Gamma}\right) \partial_{z}
$$

On the other hand

$$
\partial_{z} \log \operatorname{det}\left(I-z A_{\Gamma}\right)=\sum_{i=1}^{n} \partial_{z} \log \left(1-k_{i} z\right)=-\sum_{i=1}^{n} \frac{k_{i}}{1-k_{i} z} .
$$

By the mean curvature $H_{\Gamma}(y)$ of $\Gamma$ at $y$ we designate the trace of the second fundamental form, namely

$$
H_{\Gamma}(y)=\sum_{i=1}^{n} k_{i}(y)
$$

Similarly one can compute the mean curvature $H_{\Gamma^{z}}$ of the manifold $\Gamma^{z}$ as the sum of the eigenvalues of the matrix

$$
A_{\Gamma^{z}}=-\left[(\mathrm{Y}+z \mathrm{~B})^{T}(\mathrm{Y}+z \mathrm{~B})\right]^{-1}(\mathrm{Y}+z \mathrm{~B})^{T} \mathrm{~B} .
$$

It is easy to check

$$
A_{\Gamma^{z}}=-\left[I-z A_{\Gamma}\right]^{-1} A_{\Gamma}
$$

from where we get

$$
H_{\Gamma z}(y)=\sum_{i=1}^{n} \frac{k_{i}}{1-k_{i} z}
$$

and (2.2) holds.

Thus we have found

$$
\Delta=\partial_{z z}+\Delta_{\Gamma^{z}}-H_{\Gamma^{z}}(y) \partial_{z},
$$

that is the validity of (2.1). For later reference, let us expand

$$
H_{\Gamma^{z}}(y)=H_{\Gamma}(y)+z\left|A_{\Gamma}(y)\right|^{2}+z^{2} \sum_{i=1}^{N} k_{i}^{3}+\cdots
$$

where

$$
\underbrace{H_{\Gamma}=\sum_{i=1}^{8} k_{i}}_{\text {mean curvature }}, \quad \underbrace{\left|A_{\Gamma}\right|^{2}=\sum_{i=1}^{8} k_{i}^{2}}_{\text {norm second fundamental form }} .
$$




\subsection{Preliminaries II: Minimal hypersurface and its Jacobi operator}

The area of a portion of the surface $\Gamma$ is simply represented by the quantity

$$
\mathcal{A}[\Gamma]=\int \sqrt{[\operatorname{det} g(0)]} d \mathrm{y}
$$

integrated on a bounded region which we do not specify.

Let us consider a small normal perturbation of $\Gamma^{h}$ of $\Gamma$ (a normal graph over $\Gamma)$, described as

$$
y_{h}=y+h(y) \nu(y), \quad y \in \Gamma
$$

where $h$ is a small smooth function on $\Gamma$.

We expand in $h$ the quantity $\mathcal{A}\left[\Gamma^{h}\right]$. The metric $g(h)$ is described in coordinates $\mathrm{y}$ as

$$
g(h)=g(0)\left[I-h A_{\Gamma}\right]^{2}\left(I_{n}+\left[I-h A_{\Gamma}\right]^{-2} g(0)^{-1}(D h)^{T} D h\right) .
$$

Neglecting terms cubic in $h$ (or $D h$ ) we then find

$$
\sqrt{\operatorname{det} g(h)}=\sqrt{\operatorname{det} g(0)} \prod_{i=1}^{n}\left(1-k_{i} h\right) \sqrt{\operatorname{det}\left[I+g(0)^{-1}(D h)^{T} D h\right]}+O\left(h^{3}\right) .
$$

We compute

$$
\operatorname{det}\left[I+g(0)^{-1}(D h)^{T} D h\right]=1+\left[g(0)^{-1}(D h)^{T} D h\right]_{i i}=1+g^{i j} \partial_{j} h \partial_{i} h .
$$

On the other hand,

$$
\begin{aligned}
\log \prod_{i=1}^{n}\left(1-k_{i} h\right) & =\sum_{i} \log \left(1-k_{i} h\right) \\
& =\sum_{i}\left(-k_{i} h-\frac{1}{2} k_{i}^{2} h^{2}+O\left(h^{3}\right)\right) \\
& =-H_{\Gamma} h+\frac{1}{2}\left|A_{\Gamma}\right|^{2} h^{2}+O\left(h^{3}\right) .
\end{aligned}
$$

Thus

$$
\begin{aligned}
\prod_{i=1}^{n}\left(1-k_{i} h\right) & =\exp \left(-H_{\Gamma} h-\frac{1}{2}\left|A_{\Gamma}\right|^{2} h^{2}+O\left(h^{3}\right)\right) \\
& =1-H_{\Gamma} h-\frac{1}{2}\left|A_{\Gamma}\right|^{2} h^{2}+\frac{1}{2}\left|H_{\Gamma}\right|^{2} h^{2}+O\left(h^{3}\right) .
\end{aligned}
$$

Hence

$$
\begin{aligned}
\sqrt{\operatorname{det} g(h)}= & \sqrt{\operatorname{det} g(0)}\left(1-H_{\Gamma} h+\frac{1}{2}\left|A_{\Gamma}\right|^{2} h^{2}+\frac{1}{2}\left|H_{\Gamma}\right|^{2} h^{2}\right) \\
& \times\left(1+\frac{1}{2} g^{i j} \partial_{j} h \partial_{i} h\right)+O\left(h^{3}\right) .
\end{aligned}
$$

Thus we find

$$
\sqrt{\operatorname{det} g(h)}=\sqrt{\operatorname{det} g(0)}\left(1-H_{\Gamma} h-\frac{1}{2}\left|A_{\Gamma}\right|^{2} h^{2}+\frac{1}{2}\left|H_{\Gamma}\right|^{2} h^{2}+\frac{1}{2} g^{i j} \partial_{j} h \partial_{i} h+O\left(h^{3}\right)\right.
$$




$$
\begin{aligned}
\mathcal{A}\left[\Gamma^{h}\right]= & \mathcal{A}[\Gamma]-\int H_{\Gamma} h \sqrt{\operatorname{det} g(0)} d \mathrm{y}+\frac{1}{2} \int g^{i j}(0) \partial_{j} h \partial_{i} h-\left|A_{\Gamma}\right|^{2} h^{2} \sqrt{\operatorname{det} g(0)} d \mathrm{y} \\
& +\frac{1}{2} \int\left|H_{\Gamma}\right|^{2} h^{2} \sqrt{\operatorname{det} g(0)} d \mathrm{y}+O\left(h^{3}\right)
\end{aligned}
$$

Setting $\sqrt{\operatorname{det} g(0)} d \mathrm{y}=d \sigma(y)$ we get

$$
\mathcal{A}\left(\Gamma^{h}\right)=\mathcal{A}[\Gamma]-\int H_{\Gamma} h d \sigma-\frac{1}{2} \int\left(\Delta_{\Gamma} h+\left(\left|A_{\Gamma}\right|^{2}-\left|H_{\Gamma}\right|^{2}\right) h\right) h d \sigma+O\left(h^{3}\right) .
$$

Thus, we obtain the formulas for the first and second variations of area with respect to normal perturbations.

$$
\begin{gathered}
\delta \mathcal{A}(\Gamma)[h]:=\left.\frac{d}{d t} \mathcal{A}\left(\Gamma^{t h}\right)\right|_{t=0}=-\int H_{\Gamma} h d \sigma(y) \\
\delta^{2} \mathcal{A}(\Gamma)[h, h]:=\left.\frac{d^{2}}{d t^{2}} \mathcal{A}\left(\Gamma^{t h}\right)\right|_{t=0}=-\int\left(\Delta_{\Gamma} h+\left|A_{\Gamma}\right|^{2} h-\left|H_{\Gamma}\right|^{2}\right) h d \sigma(y) .
\end{gathered}
$$

$\Gamma$ is a minimal surface if $\delta \mathcal{A}(\Gamma)=0$, or

$$
H_{\Gamma}(y)=\sum_{i=1}^{n} k_{i}(y)=0 \text { for all } y \in \Gamma
$$

If $\Gamma$ is minimal then

$$
\delta^{2} \mathcal{A}(\Gamma)[h, g]=\int \mathcal{J}_{\Gamma}[h] g d \sigma
$$

where $\mathcal{J}_{\Gamma}$ is the Jacobi operator

$$
\mathcal{J}_{\Gamma}[h]=\Delta_{\Gamma} h+\left|A_{\Gamma}\right|^{2} h
$$

\subsection{Preliminaries III: Mean curvature and its linearization in the case of a graph}

Let us consider the special case in which $\Gamma$ is parametrized as graph,

$$
y(\mathrm{y})=\left[\begin{array}{c}
\mathrm{y} \\
F(\mathrm{y})
\end{array}\right], \quad \mathrm{Y}=\left[\begin{array}{c}
I_{n} \\
D F(\mathrm{y})
\end{array}\right]
$$

Then

$$
g(0)=\mathrm{Y}^{T} \mathrm{Y}=I_{n}+D F(\mathrm{y})^{T} D F(\mathrm{y}) .
$$

Then we easily compute

$$
\operatorname{det}(g(0))=\operatorname{det}\left(I_{n}+D F(\mathrm{y})^{T} D F(\mathrm{y})\right)=1+\sum_{i=1}^{n}\left|\partial_{i} F\right|^{2}
$$

or

$$
d \sigma(\mathrm{y})=\sqrt{1+|D F|^{2}} d \mathrm{y}
$$

Hence

$$
\mathcal{A}(F)=\int \sqrt{1+|D F|^{2}} d \mathrm{y} .
$$


Variations of area along vertical perturbations of $F$

$$
\delta \mathcal{A}(F)[\phi]:=\left.\frac{d}{d t} \mathcal{A}(F+t \phi)\right|_{t=0}=-\int \nabla \cdot\left(\frac{\nabla F}{\sqrt{1+|\nabla F|^{2}}}\right) \phi d y
$$

and

$$
\delta^{2} \mathcal{A}(F)[\phi, \phi]:=\left.\frac{d^{2}}{d t^{2}} \mathcal{A}(F+t \phi)\right|_{t=0}=-\int \nabla \cdot\left(\frac{\nabla \phi}{\sqrt{1+|\nabla F|^{2}}}-\frac{(\nabla F \cdot \nabla \phi) \nabla F}{\left(1+|\nabla F|^{2}\right)^{\frac{3}{2}}}\right) \phi d \mathrm{y} .
$$

For certain normal perturbation $\tilde{h}(t)$ with $\tilde{h}(0)=0$ we have

$$
\mathcal{A}(F+t \phi)=\mathcal{A}\left(\Gamma^{\tilde{h}(t)}\right) .
$$

Thus writing $h=\dot{\tilde{h}}(0)$ we find

$$
\delta \mathcal{A}(F)[\phi]=\delta \mathcal{A}(\Gamma)[h] .
$$

Given $\mathrm{y}$, then for certain $\tilde{\mathrm{y}}(t)$ with $\tilde{\mathrm{y}}(0)=\mathrm{y}$ we have

$$
\left[\begin{array}{c}
\tilde{\mathrm{y}}(t) \\
F(\tilde{\mathrm{y}}(t))
\end{array}\right]+\tilde{h}(t) \nu(\tilde{\mathrm{y}}(t))=\left[\begin{array}{c}
\mathrm{y} \\
F(\mathrm{y})+t \phi(\mathrm{y})
\end{array}\right] .
$$

Differentiating in $t$ at $t=0$ and taking dot product with $\nu(y)$ we find

$$
h=\dot{\tilde{h}}(0)=\nu_{n+1} \phi
$$

or

$$
\phi=\sqrt{1+|\nabla F|^{2}} h .
$$

Thus

Hence

$$
\int H_{\Gamma} h d \sigma=\int \nabla \cdot\left(\frac{\nabla F}{1+|\nabla F|^{2}}\right) \phi d \mathrm{y}
$$

$$
H_{\Gamma}=\nabla \cdot\left(\frac{\nabla F}{\sqrt{1+|\nabla F|^{2}}}\right)
$$

Similarly, since

$$
\delta^{2} \mathcal{A}(F)[\phi, \phi]=\delta^{2} \mathcal{A}(\Gamma)[h, h]+\delta \mathcal{A}(\Gamma)[\ddot{\tilde{h}}(0)]
$$

it follows that if $\Gamma$ is a minimal surface then setting

$$
L_{F}[\phi]=\nabla \cdot\left(\frac{\nabla \phi}{\sqrt{1+|\nabla F|^{2}}}-\frac{(\nabla F \cdot \nabla \phi) \nabla F}{\left(1+|\nabla F|^{2}\right)^{\frac{3}{2}}}\right)
$$

we get the identity

$$
L_{F}[\phi]=\mathcal{J}_{\Gamma}[h], \quad \phi=\sqrt{1+|\nabla F|^{2}} h .
$$

Letting $f(u)=u-u^{3}$ the equation

$$
\Delta u+f(u)=0 \quad \text { in } \mathbb{R}^{9}
$$


becomes, for

$$
u(y, \zeta):=u(x), \quad x=y+\zeta \nu(\varepsilon y), \quad y \in \Gamma_{\varepsilon},|\zeta|<\delta / \varepsilon,
$$

$\nu$ unit normal to $\Gamma$ with $\nu_{9}>0$,

$$
S(u):=\Delta u+f(u)=\Delta_{\Gamma_{\varepsilon}^{\zeta}} u-\varepsilon H_{\Gamma^{\varepsilon \zeta}}(\varepsilon y) \partial_{\zeta} u+\partial_{\zeta}^{2} u+f(u)=0 .
$$

- We look for a solution of the form (near $\Gamma_{\varepsilon}$ )

$$
u_{\varepsilon}(x)=w(\zeta-\varepsilon h(\varepsilon y))+\phi, \quad x=y+\zeta \nu(\varepsilon y)
$$

for a function $h$ defined on $\Gamma$, left as a parameter to be adjusted and $\phi$ small.

- Let $r\left(y^{\prime}, y_{9}\right)=\left|y^{\prime}\right|$. We assume a priori on $h$ that

$$
\left\|\left(1+r^{3}\right) D_{\Gamma}^{2} h\right\|_{L^{\infty}(\Gamma)}+\left\|\left(1+r^{2}\right) D_{\Gamma} h\right\|_{L^{\infty}(\Gamma)}+\|(1+r) h\|_{L^{\infty}(\Gamma)} \leq M
$$

for some large, fixed number $M$.

Let us change variables to $t=\zeta-\varepsilon h(\varepsilon y)$, or

$$
u(y, t):=u(x) \quad x=y+(t+\varepsilon h(\varepsilon y)) \nu(\varepsilon y) .
$$

The equation becomes

$$
\begin{aligned}
S(u)= & \partial_{t t} u+\Delta_{\Gamma_{\varepsilon}^{\zeta}} u-\varepsilon H_{\Gamma^{\varepsilon \zeta}}(\varepsilon y) \partial_{t} u+\varepsilon^{4}\left|\nabla_{\Gamma^{\varepsilon \zeta}} h(\varepsilon y)\right|^{2} \partial_{t t} u \\
& -2 \varepsilon^{3}\left\langle\nabla_{\Gamma^{\varepsilon \zeta}} h(\varepsilon y), \partial_{t} \nabla_{\Gamma^{\varepsilon} \zeta} u\right\rangle-\varepsilon^{3} \Delta_{\Gamma^{\varepsilon \zeta}} h(\varepsilon y) \partial_{t} u+f(u)=0, \\
& \zeta=t+\varepsilon h(\varepsilon y) .
\end{aligned}
$$

Look for solution $u_{\varepsilon}$ of the form

$$
u_{\varepsilon}(t, y)=w(t)+\phi(t, y)
$$

for a small function $\phi$.

The equation in terms of $\phi$ becomes

$$
\partial_{t t} \phi+\Delta_{\Gamma_{\varepsilon}} \phi+B \phi+f^{\prime}(w(t)) \phi+N(\phi)+E=0,
$$

where $B$ is a small linear second order operator, and

$$
E=S(w(t)), \quad N(\phi)=f(w+\phi)-f(w)-f^{\prime}(w) \phi \approx f^{\prime \prime}(w) \phi^{2} .
$$

The error of approximation is then given by the quantity

$$
E:=S(w(t))=\varepsilon^{4}\left|\nabla_{\Gamma^{\varepsilon} \zeta} h(\varepsilon y)\right|^{2} w^{\prime \prime}(t)-\left[\varepsilon^{3} \Delta_{\Gamma^{\varepsilon \zeta}} h(\varepsilon y)+\varepsilon H_{\Gamma^{\varepsilon \zeta}}(\varepsilon y)\right] w^{\prime}(t),
$$

and

$$
\varepsilon H_{\Gamma^{\varepsilon \zeta}}(\varepsilon y)=\varepsilon^{2}(t+\varepsilon h(\varepsilon y))\left|A_{\Gamma}(\varepsilon y)\right|^{2}+\varepsilon^{3}(t+\varepsilon h(\varepsilon y))^{2} \sum_{i=1}^{8} k_{i}^{3}(\varepsilon y)+\cdots .
$$

A crucial fact for estimating the size of this error is the following result by L. Simon [34] (1989): $k_{i}=O\left(r^{-1}\right)$ as $r \rightarrow+\infty$. In particular

$$
|E(y, t)| \leq C \varepsilon^{2} r(\varepsilon y)^{-2} \text {. }
$$


A fact we have to take care of is that equation

$$
\partial_{t t} \phi+\Delta_{\Gamma_{\varepsilon}} \phi+B \phi+f^{\prime}(w(t)) \phi+N(\phi)+E=0 .
$$

makes sense only for $|t|<\delta \varepsilon^{-1}$. A gluing procedure reduces the full problem to

$$
\partial_{t t} \phi+\Delta_{\Gamma_{\varepsilon}} \phi+B \phi+f^{\prime}(w) \phi+N(\phi)+E=0 \quad \text { in } \mathbb{R} \times \Gamma_{\varepsilon},
$$

where $E$ and $B$ are the same as before, but cut-off far away. $N$ is modified by the addition of a small nonlocal operator of $\phi$.

We find a small solution to Problem (2.3) in two steps which constitute an Infinite dimensional Lyapunov-Schmidt reduction:

Step 1: Given the parameter function $h$, find a a solution $\phi=\Phi(h)$ to the problem

$$
\begin{array}{r}
\partial_{t t} \phi+\Delta_{\Gamma_{\varepsilon}} \phi+B \phi+f^{\prime}(w(t)) \phi+N(\phi)+E=c(y) w^{\prime}(t) \quad \text { in } \mathbb{R} \times \Gamma_{\varepsilon}, \\
\int_{\mathbb{R}} \phi(t, y) w^{\prime}(t) d t=0 \text { for all } y \in \Gamma_{\varepsilon} .
\end{array}
$$

Step 2: Find a function $h$ such that for all $y \in \Gamma_{\varepsilon}$,

$$
c(y):=\frac{1}{\int_{\mathbb{R}} w^{\prime 2} d t} \int_{\mathbb{R}}(E+B \Phi(h)+N(\Phi(h))) w^{\prime} d t=0 .
$$

For Step 1 we solve first the linear problem

$$
\begin{gathered}
\partial_{t t} \phi+\Delta_{\Gamma_{\varepsilon}} \phi+f^{\prime}(w(t)) \phi=g(t, y)-c(y) w^{\prime}(t) \quad \text { in } \mathbb{R} \times \Gamma_{\varepsilon} \\
\int_{\mathbb{R}} \phi(y, t) w^{\prime}(t) d t=0 \quad \text { in } \Gamma_{\varepsilon}, c(y):=\frac{\int_{\mathbb{R}} g(y, t) w^{\prime}(t) d t}{\int_{\mathbb{R}} w^{\prime 2} d t} .
\end{gathered}
$$

There is a unique bounded solution $\phi:=A(g)$ if $g$ is bounded. Moreover, for any $\nu \geq 0$ we have

$$
\left\|\left(1+r(\varepsilon y)^{\nu}\right) \phi\right\|_{\infty} \leq C\left\|(1+r(\varepsilon y))^{\nu} g\right\|_{\infty} .
$$

$\Gamma_{\varepsilon} \approx \mathbb{R}^{N-1}$ around each of its points as $\varepsilon \rightarrow 0$, in uniform way. The problem is qualitatively similar to $\Gamma_{\varepsilon}$ replaced with $\mathbb{R}^{N-1}$.

Fact: The linear model problem

$$
\begin{gathered}
\partial_{t t} \phi+\Delta_{y} \phi+f^{\prime}(w(t)) \phi=g(t, y)-c(y) w^{\prime}(t) \quad \text { in } \mathbb{R}^{N} \\
\int_{\mathbb{R}} \phi(y, t) w^{\prime}(t) d t=0 \quad \text { in } \mathbb{R}^{N-1}, \quad c(y):=\frac{\int_{\mathbb{R}} g(y, t) w^{\prime}(t) d t}{\int_{\mathbb{R}} w^{\prime 2} d t}
\end{gathered}
$$

has a unique bounded solution $\phi$ if $g$ is bounded, and

$$
\|\phi\|_{\infty} \leq C\|g\|_{\infty}
$$

Let us prove first the a priori estimate: If the a priori estimate did not hold, there would exist

$$
\begin{gathered}
\left\|\phi_{n}\right\|_{\infty}=1, \quad\left\|g_{n}\right\|_{\infty} \rightarrow 0 \\
\partial_{t t} \phi_{n}+\Delta_{y} \phi_{n}+f^{\prime}(w(t)) \phi_{n}=g_{n}(t, y), \quad \int_{\mathbb{R}} \phi_{n}(y, t) w^{\prime}(t) d t=0 .
\end{gathered}
$$


Using maximum principle and local elliptic estimates, we may assume that $\phi_{n} \rightarrow$ $\phi \neq 0$ uniformly over compact sets where

$$
\partial_{t t} \phi+\Delta_{y} \phi+f^{\prime}(w(t)) \phi=0, \quad \int_{\mathbb{R}} \phi(y, t) w^{\prime}(t) d t=0 .
$$

Claim: $\phi=0$, which is a contradiction.

To establish this claim, a basic one-dimensional fact is needed: the spectral gap estimate. Let

$$
L_{0}(p):=p^{\prime \prime}+f^{\prime}(w(t)) p .
$$

Then there is a $\gamma>0$ such that if $p \in H^{1}(\mathbb{R})$ and $\int_{\mathbb{R}} p w^{\prime} d t=0$ then

$$
-\int_{\mathbb{R}} L_{0}(p) p d t=\int_{\mathbb{R}}\left(\left|p^{\prime}\right|^{2}-f^{\prime}(w) p^{2}\right) d t \geq \gamma \int_{\mathbb{R}} p^{2} d t .
$$

Using maximum principle we find $|\phi(y, t)| \leq C e^{-|t|}$. Set $\varphi(y)=\int_{\mathbb{R}} \phi^{2}(y, t) d t$. Then

$$
\begin{aligned}
\Delta_{y} \varphi(y) & =2 \int_{\mathbb{R}} \phi \Delta \phi(y, t) d t+2 \int_{\mathbb{R}}\left|\nabla_{y} \phi(y, t)\right|^{2} d t \\
& \geq-2 \int_{\mathbb{R}} \phi \partial_{t t} \phi+f^{\prime}(w) \phi^{2} d t=2 \int_{\mathbb{R}}\left(\left|\phi_{t}\right|^{2}-f^{\prime}(w) \phi^{2}\right) d t \geq \gamma \varphi(y) .
\end{aligned}
$$

Note that

$$
-\Delta_{y} \varphi(y)+\gamma \varphi(y) \leq 0, \text { and } \varphi \geq 0 \text { bounded, }
$$

implies $\varphi \equiv 0$, hence $\phi=0$, a contradiction. This proves the a priori estimate.

Existence: take $g$ compactly supported. Set $H$ be the space of all $\phi \in H^{1}\left(\mathbb{R}^{N}\right)$ with

$$
\int_{\mathbb{R}} \phi(y, t) w^{\prime}(t) d t=0 \quad \text { for all } \quad y \in \mathbb{R}^{N-1} .
$$

$H$ is a closed subspace of $H^{1}\left(\mathbb{R}^{N}\right)$.

The problem: $\phi \in H$ and

$$
\partial_{t t} \phi+\Delta_{y} \phi+f^{\prime}(w(t)) \phi=g(t, y)-w^{\prime}(t) \frac{\int_{\mathbb{R}} g(y, \tau) w^{\prime}(\tau) d \tau}{\int_{\mathbb{R}} w^{\prime 2} d \tau},
$$

can be written variationally as that of minimizing in $H$ the energy

$$
I(\phi)=\frac{1}{2} \int_{\mathbb{R}^{N}}\left|\nabla_{y} \phi\right|^{2}+\left|\phi_{t}\right|^{2}-f^{\prime}(w) \phi^{2}+\int_{\mathbb{R}^{N}} g \phi .
$$

$I$ is coercive in $H$ thanks to the $1 \mathrm{~d}$ spectral gap estimate. Existence in the general case follows by the $L^{\infty}$-a priori estimate and approximations.

We write the problem of Step 1,

$$
\begin{array}{r}
\partial_{t t} \phi+\Delta_{\Gamma_{\varepsilon}} \phi+B \phi+f^{\prime}(w(t)) \phi+N(\phi)+E=c(y) w^{\prime}(t) \quad \text { in } \mathbb{R} \times \Gamma_{\varepsilon}, \\
\int_{\mathbb{R}} \phi(t, y) w^{\prime}(t) d t=0 \quad \text { for all } y \in \Gamma_{\varepsilon},
\end{array}
$$

in fixed point form

$$
\phi=A(B \phi+N(\phi)+E) .
$$


The contraction mapping principle implies the existence of a unique solution $\phi:=$ $\Phi(h)$ with

$$
\left\|\left(1+r^{2}(\varepsilon y)\right) \phi\right\|_{\infty}=O\left(\varepsilon^{2}\right) .
$$

Finally, we carry out Step 2. We need to find $h$ such that

$$
\int_{\mathbb{R}}[E+B \Phi(h)+N(\Phi(h))]\left(\varepsilon^{-1} y, t\right) w^{\prime}(t) d t=0 \forall y \in \Gamma .
$$

Since

$$
\begin{aligned}
-E\left(\varepsilon^{-1} y, t\right)= & \varepsilon^{2} t w^{\prime}(t)\left|A_{\Gamma}(y)\right|^{2}+\varepsilon^{3}\left[\Delta_{\Gamma} h(y)+\left|A_{\Gamma}(y)\right|^{2} h(y)\right] w^{\prime}(t) \\
& +\varepsilon^{3} t^{2} w^{\prime}(t) \sum_{j=1}^{8} k_{j}(y)^{3}+\text { smaller terms }
\end{aligned}
$$

the problem becomes

$$
\mathcal{J}_{\Gamma}(h):=\Delta_{\Gamma} h+\left|A_{\Gamma}\right|^{2} h=c \sum_{i=1}^{8} k_{i}^{3}+\mathcal{N}(h) \quad \text { in } \Gamma,
$$

where $\mathcal{N}(h)$ is a small operator.

Fact: Let $0<\sigma<1$. Then if

$$
\left\|\left(1+r^{4+\sigma}\right) g\right\|_{L^{\infty}(\Gamma)}<+\infty
$$

there is a unique solution $h=T(\mathrm{~g})$ to the problem

$$
\mathcal{J}_{\Gamma}[h]:=\Delta_{\Gamma} h+\left|A_{\Gamma}(y)\right|^{2} h=\mathrm{g}(y) \quad \text { in } \Gamma
$$

with

$$
\left\|(1+r)^{2+\sigma} h\right\|_{L^{\infty}(\Gamma)} \leq C\left\|(1+r)^{4+\sigma} \mathrm{g}\right\|_{L^{\infty}(\Gamma)} .
$$

We want to solve

$$
\mathcal{J}_{\Gamma}(h):=\Delta_{\Gamma} h+\left|A_{\Gamma}\right|^{2} h=c \sum_{i=1}^{8} k_{i}^{3}+\mathcal{N}(h) \quad \text { in } \Gamma,
$$

using a fixed point formulation for the operator $T$ above. $\operatorname{In} \mathcal{N}(h)$ everything decays $O\left(r^{-4-\sigma}\right)$, but we only have

$$
\sum_{i=1}^{8} k_{i}^{3}=O\left(r^{-3}\right)
$$

\section{Two more facts:}

- There is a function $p$ smooth, with $p\left(\frac{\pi}{2}-\theta\right)=-p(\theta)$ for all $\theta \in\left(0, \frac{\pi}{4}\right)$ such that

$$
\sum_{i=1}^{8} k_{i}(y)^{3}=\frac{p(\theta)}{r^{3}}+O\left(r^{-4-\sigma}\right) .
$$


- There exists a smooth function $h_{0}(r, \theta)$ such that $h_{0}=O\left(r^{-1}\right)$ and for some $\sigma>0$

$$
\begin{gathered}
\mathcal{J}_{\Gamma}\left[h_{0}\right]=\frac{p(\theta)}{r^{3}}+O\left(r^{-4-\sigma}\right) \quad \text { as } r \rightarrow+\infty, \\
\mathcal{J}_{\Gamma}(h):=\Delta_{\Gamma} h+\left|A_{\Gamma}\right|^{2} h=c \sum_{i=1}^{8} k_{i}^{3}+O\left(r^{-4-\sigma}\right) \quad \text { in } \Gamma .
\end{gathered}
$$

Our final problem then becomes $h=h_{0}+h_{1}$ where

$$
h_{1}=T\left(O\left(r^{-4-\sigma}\right)+\mathcal{N}\left(h_{0}+h_{1}\right)\right)
$$

which we can solve for $h_{1}=O\left(r^{-2-\sigma}\right)$, using contraction mapping principle, keeping track of Lipschitz dependence in $h$ of the objects involved in in $\mathcal{N}(h)$.

As we have discussed, the Jacobi operator

$$
\mathcal{J}_{\Gamma}[h]=\Delta_{\Gamma} h+\left|A_{\Gamma}(y)\right|^{2} h,
$$

is the linearization of the mean curvature, when normal perturbations are considered. In the case of a minimal graph $x_{9}=F\left(x^{\prime}\right)$, if we linearize along vertical perturbations we get

$$
H^{\prime}(F)[\phi]=\nabla \cdot\left(\frac{\nabla \phi}{\sqrt{1+|\nabla F|^{2}}}-\frac{(\nabla F \cdot \nabla \phi)}{\left(1+|\nabla F|^{2}\right)^{\frac{3}{2}}} \nabla F\right) .
$$

As we have seen, these two operators are linked through the relation

$$
\mathcal{J}_{\Gamma}[h]=H^{\prime}(F)[\phi], \quad \text { where } \quad \phi\left(x^{\prime}\right)=\sqrt{1+\left|\nabla F\left(x^{\prime}\right)\right|^{2}} h\left(x^{\prime}, F\left(x^{\prime}\right)\right) .
$$

Next we discuss the proofs of the facts used above:

1. If $g=O\left(r^{-4-\sigma}\right)$ there is a unique solution to $\mathcal{J}_{\Gamma}[h]=g$ with

$$
\left\|(1+r)^{2+\sigma} h\right\|_{L^{\infty}(\Gamma)} \leq C\left\|(1+r)^{4+\sigma} \mathrm{g}\right\|_{L^{\infty}(\Gamma)} .
$$

2. There is a function $p$ smooth, with $p\left(\frac{\pi}{2}-\theta\right)=-p(\theta)$ for all $\theta \in\left(0, \frac{\pi}{4}\right)$ such that

$$
\sum_{i=1}^{8} k_{i}(y)^{3}=\frac{p(\theta)}{r^{3}}+O\left(r^{-4-\sigma}\right) .
$$

3. There exists $h_{0}(r, \theta)$ such that $h_{0}=O\left(r^{-1}\right)$ and

$$
\mathcal{J}_{\Gamma}\left[h_{0}\right]=\frac{p(\theta)}{r^{3}}+O\left(r^{-4-\sigma}\right) \quad \text { as } r \rightarrow+\infty .
$$

The closeness between $\mathcal{J}_{\Gamma_{0}}$ and $\mathcal{J}_{\Gamma}$. The following statement is proven in [16].

Let $p \in \Gamma$ with $r(p) \gg 1$. There is a unique $\pi(p) \in \Gamma_{0}$ such that $\pi(p)=p+t_{p} \nu(p)$. Let us assume

Then

$$
\tilde{h}(\pi(y))=h(y), \quad \text { for all } \quad y \in \Gamma, \quad r(y)>r_{0}
$$

$$
\mathcal{J}_{\Gamma}[h](y)=\left[\mathcal{J}_{\Gamma_{0}}\left[h_{0}\right]+O\left(r^{-2-\sigma}\right) D_{\Gamma_{0}}^{2} h_{0}+O\left(r^{-3-\sigma}\right) D_{\Gamma_{0}} h_{0}+O\left(r^{-4-\sigma}\right) h_{0}\right](\pi(y)) .
$$


The operator $\mathcal{J}_{\Gamma_{0}}[h]$ does not exactly coincide with $H^{\prime}\left(F_{0}\right)\left[\sqrt{1+\left|\nabla F_{0}\right|^{2}} h\right]$ but they differ only on terms with very rapid decay. This allows us to make explicit computations for approximate solutions and barriers.

We compute explicitly

$$
\begin{aligned}
H^{\prime}\left(F_{0}\right)[\phi]= & \frac{1}{r^{7} \sin ^{3}(2 \theta)}\left\{\left(9 g^{2} \tilde{w} r^{3} \phi_{\theta}\right)_{\theta}+\left(r^{5} g^{\prime 2} \tilde{w} \phi_{r}\right)_{r}\right. \\
& \left.-3\left(g g^{\prime} \tilde{w} r^{4} \phi_{r}\right)_{\theta}-3\left(g g^{\prime} \tilde{w} r^{4} \phi_{\theta}\right)_{r}\right\} \\
& +\frac{1}{r^{7} \sin ^{3}(2 \theta)}\left\{\left(r^{-1} \tilde{w} \phi_{\theta}\right)_{\theta}+\left(r \tilde{w} \phi_{r}\right)_{r}\right\},
\end{aligned}
$$

where

$$
\tilde{w}(r, \theta):=\frac{\sin ^{3} 2 \theta}{\left(r^{-4}+9 g^{2}+g^{\prime 2}\right)^{\frac{3}{2}}} .
$$

Further expand

$$
L[\phi]:=H^{\prime}\left(F_{0}\right)[\phi]:=L_{0}+L_{1},
$$

with

$$
\begin{aligned}
L_{0}[\phi]= & \frac{1}{r^{7} \sin ^{3}(2 \theta)}\left\{\left(9 g^{2} \tilde{w}_{0} r^{3} \phi_{\theta}\right)_{\theta}+\left(r^{5} g^{\prime 2} \tilde{w}_{0} \phi_{r}\right)_{r}\right. \\
& \left.-3\left(g g^{\prime} \tilde{w}_{0} r^{4} \phi_{r}\right)_{\theta}-3\left(g g^{\prime} \tilde{w}_{0} r^{4} \phi_{\theta}\right)_{r}\right\} \\
& +\frac{1}{r^{7} \sin ^{3}(2 \theta)}\left\{\left(r^{-1} \tilde{w}_{0} \phi_{\theta}\right)_{\theta}+\left(r \tilde{w}_{0} \phi_{r}\right)_{r}\right\}
\end{aligned}
$$

where

$$
\tilde{w}_{0}(\theta):=\frac{\sin ^{3} 2 \theta}{\left(9 g^{2}+g^{\prime 2}\right)^{\frac{3}{2}}} .
$$

An important fact: If $0<\sigma<1$ there is a positive supersolution $\bar{\phi}=O\left(r^{-\sigma}\right)$ to

$$
-L[\bar{\phi}] \geq \frac{1}{r^{4+\sigma}} \quad \text { in } \Gamma \text {. }
$$

We have that

$$
L_{0}\left[r^{-\sigma} q(\theta)\right]=\frac{1}{r^{4+\sigma}} \frac{9 g^{\frac{4-\sigma}{3}}}{\sin ^{3} 2 \theta}\left[\frac{g^{\frac{2}{3}} \sin ^{3} 2 \theta}{\left(9 g^{2}+g^{\prime 2}\right)^{\frac{3}{2}}}\left(g^{\frac{\sigma}{3}} q\right)^{\prime}\right]^{\prime}=\frac{1}{r^{4+\sigma}}
$$

if and only if $q(\theta)$ solves the ODE

$$
\left[\frac{g^{\frac{2}{3}} \sin ^{3} 2 \theta}{\left(9 g^{2}+g^{2}\right)^{\frac{3}{2}}}\left(g^{\frac{\sigma}{3}} q\right)^{\prime}\right]^{\prime}=\frac{1}{9} \sin ^{3} 2 \theta g(\theta)^{-\frac{4-\sigma}{3}}, .
$$

A solution in $\left(\frac{\pi}{4}, \frac{\pi}{2}\right)$ :

$$
q(\theta)=\frac{1}{9} g^{-\frac{\sigma}{3}}(\theta) \int_{\frac{\pi}{4}}^{\theta} \frac{\left(9 g^{2}+g^{2}\right)^{\frac{3}{2}}}{g^{\frac{2}{3}} \sin ^{3}(2 s)} d s \int_{s}^{\frac{\pi}{2}} g^{-\frac{4-\sigma}{3}}(\tau) \sin ^{3}(2 \tau) d \tau
$$


Since $g^{\prime}\left(\frac{\pi}{4}\right)>0, q$ is defined up to $\frac{\pi}{4}$ and can be extended smoothly (evenly) to $\left(0, \frac{\pi}{4}\right)$. Thus and $\bar{\phi}:=q(\theta) r^{-\sigma}$ satisfies

$$
-L_{0}(\bar{\phi})=r^{-4-\mu} \quad \text { in } \mathbb{R}^{8} .
$$

We can show that also $-L(\bar{\phi}) \geq r^{-4-\sigma}$ for all large $r$. Thus

$$
-\mathcal{J}_{\Gamma_{0}}[\bar{h}] \geq r^{-4-\sigma}, \quad \bar{h}=\frac{\phi}{\sqrt{1+\left|\nabla F_{0}\right|^{2}}} \sim r^{-2-\sigma} .
$$

The closeness of $\mathcal{J}_{\Gamma}$ and $\mathcal{J}_{\Gamma_{0}}$ makes $\bar{h}$ to induce a positive supersolution $\hat{h} \sim$ $r^{-2-\sigma}$ to

$$
-\mathcal{J}_{\Gamma}[\hat{h}] \geq r^{-4-\sigma} \quad \text { in } \Gamma .
$$

We conclude by a barrier argument that Fact 1 holds: if $\left\|\left(1+r^{4+\sigma}\right) g\right\|_{L^{\infty}(\Gamma)}<+\infty$ there is a unique $h$ with $\mathcal{J}_{\Gamma}[h]=\mathrm{g}$ and

$$
\left\|(1+r)^{2+\sigma} h\right\|_{L^{\infty}(\Gamma)} \leq C\left\|(1+r)^{4+\sigma} \mathrm{g}\right\|_{L^{\infty}(\Gamma)} .
$$

Let $k_{i}^{0}(y)$ be the principal curvatures of $\Gamma_{0}$.

\section{The following hold:}

$$
\begin{array}{rr}
- & \sum_{i=1}^{8} k_{i}(y)^{3}=\sum_{i=1}^{8} k_{i}^{0}(\pi(y))^{3}+O\left(r^{-4-\sigma}\right) \\
\text { - } & \sum_{i=1}^{8} k_{i}^{0}(y)^{3}=\frac{p(\theta)}{r^{3}}+O\left(r^{-4-\sigma}\right)
\end{array}
$$

$p$ smooth, $p\left(\frac{\pi}{2}-\theta\right)=-p(\theta)$ for all $\theta \in\left(0, \frac{\pi}{4}\right)$.

We claim: there exists a smooth function $h_{*}(r, \theta)$ such that $h_{*}=O\left(r^{-1}\right)$ and for some $\sigma>0$,

$$
\mathcal{J}_{\Gamma_{0}}\left[h_{*}\right]=\frac{p(\theta)}{r^{3}}+O\left(r^{-4-\sigma}\right) \quad \text { as } r \rightarrow+\infty
$$

Setting $h_{0}(y)=h_{*}(\pi(y))$ we then get $h_{0}=O\left(r^{-1}\right)$ and

$$
\mathcal{J}_{\Gamma}(h):=\Delta_{\Gamma} h+\left|A_{\Gamma}\right|^{2} h=c \sum_{i=1}^{8} k_{i}^{3}+O\left(r^{-4-\sigma}\right) \quad \text { in } \Gamma,
$$

namely the validity of Fact 2 .

\section{Construction of $h_{*}$.}

We argue as before (separation of variables) to find $q(\theta)$ solution of

$$
\begin{gathered}
L_{0}(r q(\theta))=\frac{p(\theta)}{r^{3}}, \theta \in\left(\frac{\pi}{4}, \frac{\pi}{2}\right), \\
q(\theta)=-\frac{1}{9} g^{\frac{1}{3}}(\theta) \int_{\frac{\pi}{4}}^{\theta}\left(9 g^{2}+g^{\prime 2}\right)^{\frac{3}{2}} \frac{g^{-\frac{2}{3}} d s}{\sin ^{3}(2 s)} \int_{s}^{\frac{\pi}{2}} p(\tau) g^{-\frac{5}{3}}(\tau) \sin ^{3}(2 \tau) d \tau .
\end{gathered}
$$


Let $\eta(s)=1$ for $s<1,=0$ for $s>2$ be a smooth cut-off function. Then

$$
\phi_{0}(r, \theta):=(1-\eta(s)) r q(\theta) \quad \text { in }\left(\frac{\pi}{4}, \frac{\pi}{2}\right), \quad s=r^{2} g(\theta) .
$$

satisfies

$$
L\left(\phi_{0}\right)=\frac{p(\theta)}{r^{3}}+O\left(r^{-4-\frac{1}{3}}\right) .
$$

Finally, the function

$$
h_{*}=\frac{\phi_{0}}{\sqrt{1+\left|\nabla F_{0}\right|^{2}}}=O\left(r^{-1}\right)
$$

extended oddly through $\theta=\frac{\pi}{4}$ satisfies

$$
J_{\Gamma_{0}}\left[h_{*}\right]=\frac{p(\theta)}{r^{3}}+O\left(r^{-4-\frac{1}{3}}\right)
$$

as desired.

\section{Some further results on entire solutions}

Loosely speaking, the method of construction of solutions described so far applies to finding an entire solution $u_{\varepsilon}$ to $\Delta u+u-u^{3}=0$ with transition set near $\Gamma_{\varepsilon}=\varepsilon^{-1} \Gamma$, whenever $\Gamma$ is a minimal hypersurface embedded in $\mathbb{R}^{N}$, that splits the space into two components, and for which enough control at infinity is present to invert globally its Jacobi operator.

\subsection{An important example for $N=3$ : finite Morse index solutions}

We have the validity of the following result

Theorem 2 ([19]). Let $\Gamma$ be a complete, embedded minimal surface in $\mathbb{R}^{3}$ with finite total curvature: $\int_{\Gamma}|K|<\infty, K$ Gauss curvature.

If $\Gamma$ is non-degenerate, namely its bounded Jacobi fields originate only from rigid motions, then for small $\varepsilon>0$ there is a solution $u_{\varepsilon}$ to $(A C)$ with

$$
u_{\varepsilon}(x) \approx w(t), \quad x=y+t \nu_{\varepsilon}(y) .
$$

In addition $i\left(u_{\varepsilon}\right)=i(\Gamma)$ where $i$ denotes Morse index.

For example: nondegeneracy and Morse index are known for the catenoid and the Costa-Hoffmann-Meeks surfaces (found in [12, 26]), see (Nayatani [31] (1990), Morabito [30], (2008)).

\subsection{An example with infinite total curvature $\int_{\Gamma}|K|=\infty$}

The helicoid, is a classical embedded minimal surface whose total curvature is infinite: this surface, dependent on a parameter $\lambda$ can be described as follows.

$$
H_{\lambda}=\left\{(r \cos \theta, r \sin \theta, z) \in \mathbb{R}^{3} / z=\frac{\lambda}{\pi} \theta\right\} .
$$

The following result holds: 
Theorem 3. [20]

- If $\lambda>\pi$ There exists a solution to the Allen Cahn equation in $\mathbb{R}^{3}$ whose zero level set is exactly $H_{\lambda}$

- If $\lambda \leq \pi$ then any solution which vanishes on $H_{\lambda}$ must be identically zero.

The zero-level set of $u$ : the helicoid $z=\frac{\lambda}{\pi} \theta$.

As $r \rightarrow+\infty, v(r, s) \approx w(s)$ where $w$ is the unique solution of

$$
w^{\prime \prime}+f(w)=0, \quad w(\lambda)=0=w(0)
$$

$w \neq 0$ exists and it is unique up to translations if and only if $\lambda>\pi$.

Those found in the above result are Screw-motion invariant solutions. If $\lambda>\pi$, there exists a solution $u(r, \theta, z)$, whose zero set corresponds exactly to the helicoid $z=\frac{\lambda}{\pi} \theta$, invariant under screw motion:

$$
u(r, \theta, z)=u\left(r, \theta-\alpha, z-\frac{\lambda}{\pi} \alpha\right)=u\left(r, 0, z-\frac{\lambda}{\pi} \theta\right) \text { for all } \quad \alpha .
$$

which reduce the equation to

$$
\begin{gathered}
u(r, \theta, z) \equiv v\left(r, z-\frac{\lambda}{\pi} \theta\right), \\
v_{s s}+v_{r r}+\frac{v_{r}}{r}+\frac{\lambda^{2}}{r^{2} \pi^{2}} v_{s s}+f(v)=0, \quad v(r, 0)=0=v(r, \lambda) .
\end{gathered}
$$

\subsection{The stable De Giorgi conjecture}

A question related with De Giorgi conjecture is the following:

Is it true that a bounded, stable solution to (1.1) must have planar level sets?

Stability of a solution $u$ means that

$$
\int|\nabla \phi|^{2}+\left(1-3 u^{2}\right) \phi^{2} \geq 0 \quad \text { for all } \phi \in C_{0}^{\infty}\left(\mathbb{R}^{n}\right) .
$$

This condition is implied by the fact that $\partial_{x_{n}} u>0$ as it is standard to check. The result in [16] implies that the above assertion is false for dimension $N \geq 9$. On the other hand, the conjecture is true for $n=2$, see Dancer [13].

In [32] it is found stable solutions with non planar level sets in dimension $n=8$, using the existence of minimal cones in this dimension, and a foliation of stable minimal surfaces stemming from them.

\section{Another application of the BDG minimal graph: overdetermined semilinear equation}

Let $\Omega$ be a smooth domain, and $f$ a Lipschitz function. We consider the problem

$$
\begin{gathered}
\Delta u+f(u)=0, u>0 \quad \text { in } \Omega, u \in L^{\infty}(\Omega) \\
u=0, \quad \partial_{\nu} u=\text { constant } \text { on } \partial \Omega .
\end{gathered}
$$


Since this problem is overdetermined, it is natural to believe that solvability of such a problem may imply constraints on the geometry of the domain.

Serrin (1971) proved that if $\Omega$ is bounded and there is a solution to (4.1) then $\Omega$ must be a ball.

Let us consider now the case of an entire epigraph

$$
\Omega=\left\{\left(x^{\prime}, x_{N}\right) / x^{\prime} \in \mathbb{R}^{N-1}, x_{N}>\varphi\left(x^{\prime}\right)\right\}, \quad \Gamma=\partial \Omega .
$$

- Berestycki, Caffarelli and Nirenberg [6] (1997) proved that if $\varphi$ is Lispchitz and asymptotically flat then it must be linear and $u$ depends on only one variable. They conjecture that this should be true for any arbitrary smooth function $\varphi$.

- Farina and Valdinoci [23] (2009) lifted asymptotic flatness for $N=2,3$ and for $N=4,5$ and $f(u)=u-u^{3}$.

Theorem 4 ([21]). In Dimension $N \geq 9$ there exists a solution to Problem (4.1) with $f(u)=u-u^{3}$, in an entire epigraph $\Omega$ which is not a half-space.

The proof consists of finding the region $\Omega$ for which

$$
\partial \Omega=\left\{y+\varepsilon h(\varepsilon y) \nu(\varepsilon y) / y \in \Gamma_{\varepsilon}\right\} .
$$

for $h$ a small decaying function on $\Gamma$, with $\Gamma$ a BDG graph. The construction carries over for more general surfaces $\Gamma$.

Let us set

$$
u_{0}(x)=w(t), \quad x=y+(t+\varepsilon h(\varepsilon y)) \nu(\varepsilon y) \quad \Omega=\{t>0\} .
$$

Again for $x=y+\varepsilon(t+\varepsilon h(\varepsilon y))$, we look for a solution for $t>0$ with $u(t, y)=$ $w(t)+\phi(t, x)$. Then at main order $\phi$ should satisfy

$$
\begin{gathered}
\partial_{t t} \phi+\Delta_{\Gamma_{\varepsilon}} \phi+f^{\prime}(w(t)) \phi \approx E \\
\phi(0, y)=0, \phi_{t}(0, y) \approx \alpha \quad \forall y \in \Gamma_{\varepsilon}, \\
E=\Delta u_{0}+f\left(u_{0}\right) \\
=\varepsilon^{4}\left|\nabla_{\Gamma^{\varepsilon \zeta}} h(\varepsilon y)\right|^{2} w^{\prime \prime}(t)-\left[\varepsilon^{3} \Delta_{\Gamma^{\varepsilon \zeta}} h(\varepsilon y)+\varepsilon H_{\Gamma^{\varepsilon \zeta}}(\varepsilon y)\right] w^{\prime}(t), \\
E=\varepsilon H_{\Gamma}(\varepsilon y) w^{\prime}(t)+O\left(\varepsilon^{2}\right) .
\end{gathered}
$$

Integrating the equation for $\phi$ we find

$$
-w^{\prime}(0) \phi_{t}(0, y) \approx \int_{0}^{\infty} E(y, t) w^{\prime}(t) d t=-\varepsilon H_{\Gamma}(\varepsilon y) \int_{0}^{\infty} w^{\prime}(t)^{2} d t+O\left(\varepsilon^{2}\right) .
$$

We need

$$
H_{\Gamma} \equiv H=\text { constant }
$$

Namely $\Gamma$ should be a constant mean curvature surface. Then we solve imposing $\alpha=\varepsilon\left(H / w^{\prime}(0)\right) \int_{0}^{\infty} w^{\prime}(t)^{2} d t$.

Let us assume that that $\Gamma$ is a smooth surface such that

$$
H_{\Gamma} \equiv H=\text { constant } \text {. }
$$


The approximation can be improved as follows: For $x=y+\varepsilon(t+\varepsilon h(\varepsilon y))$, we look now for a solution for $t>0$ with

$$
u(t, y)=w(t)+\phi(t, y), \quad \phi(0, y)=0 .
$$

Imposing $\alpha=\left(H / w^{\prime}(0)\right) \int_{0}^{\infty} w^{\prime}(t)^{2} d t$. we can solve

$$
\psi^{\prime \prime}+f^{\prime}(w(t)) \psi=H w^{\prime}(t), \quad t>0, \quad \psi(0)=0, \psi^{\prime}(0)=\alpha
$$

which is solvable for $\psi$ bounded. Then the approximation $u_{1}(x)=w(t)+\varepsilon \psi(t)$ produces a new error of order $\varepsilon^{2}$. And the equation for $\phi=\varepsilon \psi(t)+\phi_{1}$ now becomes

$$
\begin{gathered}
\partial_{t t} \phi_{1}+\Delta_{\Gamma_{\varepsilon}} \phi_{1}+f^{\prime}(w(t)) \phi_{1}=E_{1}=O\left(\varepsilon^{2}\right) \\
\phi_{1}(0, y)=0, \phi_{1, t}(0, y)=0 .
\end{gathered}
$$

The construction follows a scheme similar to that for the entire solution, but it is more subtle in both theories needed in Steps 1 and 2.

\section{Another application of the BDG surface: Self-translating graph solutions to mean curvature flow}

A family of graphs

$$
\Sigma(t)=\left\{(x, F(x, t)) / \quad x \in \mathbb{R}^{N}\right\} \subset \mathbb{R}^{N+1}
$$

evolves by mean curvature iff $F$ solves the graphical mean curvature flow, given by the parabolic PDE

$$
\frac{\partial F}{\partial t}=\sqrt{1+|\nabla F|^{2}} \nabla \cdot\left(\frac{\nabla F}{\sqrt{1+|\nabla F|^{2}}}\right) .
$$

Self-translating solution with speed $c$ :

$$
F(x, t)=c t+G(x)
$$

$F(x, t)=c t+F(x)$ solves (5.2) iff

$$
\nabla \cdot\left(\frac{\nabla F}{\sqrt{1+|\nabla F|^{2}}}\right)=\frac{c}{\sqrt{1+|\nabla F|^{2}}} \text { in } \mathbb{R}^{N}
$$

A Bernstein problem for (5.3):

(B) Is it true that entire solutions of (5.3) for $c \geq 0$ need to be convex?

This statement for $c=0$ reduces to Bernstein's problem: If $F$ solving (5.3) was necessarily convex, then so would be $-F$. Hence $F$ would be a linear affine function.

True for $N=2$ (X.-J. Wang [36] 2010). Solutions are radial. 
Examples of self-translating graphs:

- Altschuler and Wu [3], Clutterbuck, Schnürer and Schulze [11]: A unique radially symmetric solution (for $c=1, N \geq 2$ )

$$
F(|x|)=\frac{|x|^{2}}{2(N-1)}-\log |x|+O\left(|x|^{-1}\right) \quad \text { as }|x| \rightarrow \infty .
$$

- X.-J. Wang [36] has found examples for $N \geq 3$ of convex, non-radial solutions.

Note: For $c \neq 0 F$ solves (5.3) iff $G(x)=c F\left(c^{-1} x\right)$ solves (5.3) for $c=1$ :

$$
\begin{gathered}
\nabla \cdot\left(\frac{\nabla G}{\sqrt{1+|\nabla G|^{2}}}\right)=\frac{1}{\sqrt{1+|\nabla G|^{2}}} \text { in } \mathbb{R}^{N} . \\
x_{N+1}=F(|x|)=\frac{|x|^{2}}{2(N-1)}-\log |x|+O\left(|x|^{-1}\right) .
\end{gathered}
$$

The answer to (B) is negative for $c>0$ and $N \geq 8$, in analogy to the result of Bombieri, De Giorgi and Giusti:

Theorem 5 ([18]). Assume that $N \geq 8$. Then there exists a one-parameter family of non-convex entire solutions $F_{\varepsilon}(x), \varepsilon>0$. to Equation $(M C G)$ for $c=1$.

Replacing $F_{\varepsilon}(x)$ with $\varepsilon^{-1} F_{\varepsilon}(\varepsilon x)$ we are reduced to finding a non-convex solution $F_{\varepsilon}$ of the equation

$$
\nabla \cdot\left(\frac{\nabla F}{\sqrt{1+|\nabla F|^{2}}}\right)=\frac{\varepsilon}{\sqrt{1+|\nabla F|^{2}}} \text { in } \mathbb{R}^{N} .
$$

When $\varepsilon=0$ there is a nontrivial solution (the BDG graph) of the form

$$
\begin{gathered}
x=(\mathbf{u}, \mathbf{v}) \in \mathbb{R}^{4} \times \mathbb{R}^{4} \longmapsto \bar{F}(u, v), \quad u=|\mathbf{u}|, v=|\mathbf{v}|, \\
\bar{F}(x)=O\left(|x|^{3}\right) \quad \text { as }|x| \rightarrow \infty .
\end{gathered}
$$

For small $\varepsilon>0$ we find

$$
F_{\varepsilon}(x)=\bar{F}(x)+\varepsilon \phi_{\varepsilon}(x) .
$$

with

$$
\begin{gathered}
\left|\phi_{\varepsilon}(x)\right| \leq C\left(|x|^{2}+1\right) \quad \text { in } \mathbb{R}^{8}, \\
F_{\varepsilon}(x)=\bar{F}(x)+O\left(\varepsilon|x|^{2}\right) .
\end{gathered}
$$

The method: construction of ordered sub and super solutions for the equation

$$
M[F]:=\nabla \cdot\left(\frac{\nabla F}{\sqrt{1+|\nabla G|^{2}}}\right)-\frac{\varepsilon}{\sqrt{1+|\nabla F|^{2}}}=0 \text { in } \mathbb{R}^{8} .
$$

The equation $M[\bar{F}+\varphi]=0$ is at main order, for $r$ large,

$$
L_{\bar{F}}[\varphi]=\frac{\varepsilon}{\sqrt{1+\left|\nabla F_{0}\right|^{2}}} \approx \frac{\varepsilon p_{1}(\theta)}{r^{2}} .
$$


We can solve by barriers equations of the form

$$
L_{\bar{F}}[\varphi]=\mathrm{g}=O\left(r^{-4-\sigma}\right) .
$$

where $\sigma>0$. The barrier procedure however does not work for decays $O\left(r^{-4}\right)$ or slower, and the main error term only has decay $O\left(r^{-2}\right)$.

To overcome this difficulty, we need to improve the approximation:

There is a smooth function $\varphi_{*}(r, \theta)=O\left(\varepsilon r^{2}\right)$ as $r \rightarrow \infty$ such that for some $\sigma>0$

$$
M\left[F+\varphi_{*}\right]=O\left(r^{-4-\sigma}\right) .
$$

The function $\varphi_{*}(r, \theta)$ is found by setting first

$$
\varphi_{*}(r, \theta)=\varepsilon \varphi_{1}(r, \theta)+\varepsilon^{2} \varphi_{2}(r, \theta)+\varepsilon^{3} \varphi_{2}(r, \theta)+\cdots
$$

and solving (explicitly, up to fast decaying terms) the linear equations for the first 3 coefficients (which at main order separate variables).

This and a refinement of the asymptotic behavior of $\bar{F}-F_{0}$ yields the result.

After the above is achieved, the second step is the following.

There exists a smooth function $\phi$ with $\phi(r, \theta)=O\left(\varepsilon r^{-\sigma}\right)$ as $r \rightarrow \infty$ for some $\sigma>0$, such that globally

$$
M\left[\bar{F}+\varphi_{*}+\phi\right] \leq 0, \quad M\left[F+\varphi_{*}-\phi\right] \geq 0 .
$$

In essence, $\phi$ is a positive supersolution for the equation

$$
L_{\bar{F}}[\phi]=-M\left[\bar{F}+\varphi_{*}\right]=O\left(\varepsilon r^{-4-\sigma}\right) .
$$

Using the above fact, the proof of our main result can be concluded as follows.

We consider an arbitrary $R$ and the equation

$$
\begin{gathered}
\nabla \cdot\left(\frac{\nabla F}{\sqrt{1+|\nabla F|^{2}}}\right)-\frac{\varepsilon}{\sqrt{1+|\nabla F|^{2}}}=0 \text { in } B_{R}(0), \\
F=\bar{F}+\varphi_{*}-\phi \quad \text { on } \partial B_{R}(0) .
\end{gathered}
$$

By super-subsolutions, this problem has a smooth solution $F_{R}$ with

$$
\bar{F}+\varphi_{*}-\phi \leq F_{R} \leq \bar{F}+\varphi_{*}+\phi \quad \text { in } B_{R}(0)
$$

$G_{R}$ is increasing in $R$ on each fixed ball $B_{R_{0}}(0)$.

Regularity theory for the mean curvature operator yields $\left|\nabla F_{R}\right| \leq C\left(R_{0}\right)$ on this ball. Hence $F_{R} \rightarrow F$ in local $C^{2}$-sense. Thus $F$ solves

$$
\begin{gathered}
\nabla \cdot\left(\frac{\nabla F}{\sqrt{1+|\nabla F|^{2}}}\right)-\frac{\varepsilon}{\sqrt{1+|\nabla F|^{2}}}=0 \text { in } \mathbb{R}^{8}, \\
\bar{F}+\varphi_{*}-\phi \leq F \leq \bar{F}+\varphi_{*}+\phi \text { in } \mathbb{R}^{8} .
\end{gathered}
$$




\section{References}

[1] S.M. Allen and J.W. Cahn A microscopic theory for antiphase boundary motion and its application to antiphase domain coarsening, Acta Metall. 27 (1979), 1084-1095.

[2] F.J. Almgren Jr. Some interior regularity theorems for minimal surfaces and an extension of Bernstein's theorem., Ann. of Math. (2) 841966 277-292.

[3] S. Altschuler, L.-F. Wu, Translating surfaces of the non-parametric mean curvature flow with prescribed contact angle. Calc. Var. Partial Differential Equations 2(1), (1994), 101111.

[4] L. Ambrosio and X. Cabré, Entire solutions of semilinear elliptic equations in $\mathbb{R}^{3}$ and a conjecture of De Giorgi, Journal Amer. Math. Soc. 13 (2000), 725-739.

[5] S. Angenent, J.J.L. Velázquez, Degenerate neckpinches in mean curvature flow. J. Reine Angew. Math. 482, (1997) 15-66.

[6] H. Berestycki, L. Caffarelli, and L. Nirenberg, Further qualitative properties for elliptic equations in unbounded domains, Ann. Scuola Norm. Sup. Pisa Cl. Sci. 25 (1997), 69-94.

[7] H. Berestycki, L. Caffarelli and L. Nirenberg, Monotonicity for elliptic equations in an unbounded Lipschitz domain, Comm. Pure Appl. Math., 50(1997), 1089-1111.

[8] S. Bernstein, Sur les surfaces définies au moyen de leur curbature moyenne ou totale. Ann. Sci. Ec. Norm. Sup. 27, (1910) 233-256.

[9] E. Bombieri, E. De Giorgi, E. Giusti, Minimal cones and the Bernstein problem, Invent. Math. 7 (1969) 243-268.

[10] X. Cabré, J. Terra Saddle-shaped solutions of bistable diffusion equations in all of $\mathbb{R}^{2 m}$. J. Eur. Math. Soc. 11, Issue 4 (2009), 819-943.

[11] J. Clutterbuck, O. Schnurer and F. Schulze, Stability of translating solutions to mean curvature flow, Cal. Var. PDE 29(2007), 281-293.

[12] C.J. Costa, Imersoes minimas en $\mathbb{R}^{3}$ de genero un e curvatura total finita. PhD thesis, IMPA, Rio de Janeiro, Brasil (1982).

[13] E.N. Dancer, Stable and finite Morse index solutions on $\mathbf{R}^{n}$ or on bounded domains with small diffusion. Trans. Amer. Math. Soc. 357 (2005), no. 3, 1225-1243.

[14] E. De Giorgi, Una estensione del teorema di Bernstein. Ann. Scuola Norm. Sup. Pisa (3) $19196579-85$.

[15] E. De Giorgi, Convergence problems for functionals and operators, Proc. Int. Meeting on Recent Methods in Nonlinear Analysis (Rome, 1978), 131-188, Pitagora, Bologna (1979).

[16] M. del Pino, M. Kowalczyk, J. Wei, On De Giorgi's Conjecture in Dimensions $N \geq 9$, To appear in Ann. of Math, preprint arXiv.org 0806.3141.

[17] M. del Pino, M. Kowalczyk, J. Wei, A counterexample to a conjecture by De Giorgi in large dimensions, Comp. Rend. Mathematique 346 (2008), 23-24, 1261-1266.

[18] P. Daskalopoulos, M. del Pino, M. Kowalczyk, J. Wei, Mean convex solutions to the graphic mean curvature flow In preparation. 
[19] M. del Pino, M. Kowalczyk, J. Wei, Entire Solutions of the Allen-Cahn equation and Complete Embedded Minimal Surfaces of Finite Total Curvature in $\mathbb{R}^{3}$. Preprint arXiv.org 0902.204 2009.

[20] M. del Pino, M. Musso, F. Pacard, Soloutions of the Allen-Cahn equation invariant under screw motion. Preprint 2011 http://www.math.polytechnique.fr/ pacard/PR2011-1.pdf.

[21] M. del Pino, F. Pacard, J. Wei, Overdetermined semilinear elliptic problems: the role of minimal and constant mean curvature surfaces. In preparation.

[22] A. Farina and E. Valdinoci, The state of art for a conjecture of De Giorgi and related questions. "Reaction-Diffusion Systems and Viscosity Solutions", World Scientific, 2008.

[23] A. Farina and E. Valdinoci, Flattening results for elliptic PDEs in unbounded domains with applications to overdetermined problems. Arch. Ration. Mech. Anal. 195 (2010), no. 3, 1025-1058.

[24] W.H. Fleming, On the oriented Plateau problem. Rend. Circ. Mat. Palermo (2) 111962 69-90.

[25] N. Ghoussoub and C. Gui, On a conjecture of De Giorgi and some related problems, Math. Ann. 311 (1998), 481-491.

[26] D. Hoffman and W.H. Meeks III, A complete embedded minimal surface in $\mathbb{R}^{3}$ with genus one and three ends, J. Diff. Geom. 21 (1985), 109-127.

[27] D. Jerison and R. Monneau, Towards a counter-example to a conjecture of De Giorgi in high dimensions, Ann. Mat. Pura Appl. 183 (2004), 439-467.

[28] L. Modica, Convergence to minimal surfaces problem and global solutions of $\Delta u=$ $2\left(u^{3}-u\right)$., Proceedings of the International Meeting on Recent Methods in Nonlinear Analysis (Rome, 1978), pp. 223-244, Pitagora, Bologna, (1979).

[29] L. Modica, S. Mortola, Un esempio di $\Gamma$-convergenza., Boll. Unione Mat. Ital. Sez. B 14, 285-299 (1977).

[30] F. Morabito, Index and nullity of the Gauss map of the Costa-Hoffman-Meeks surfaces, preprint 2008.

[31] S. Nayatani, Morse index and Gauss maps of complete minimal surfaces in Euclidean 3-space, Comm. Math. Helv. 68(4)(1993), 511-537.

[32] F. Pacard, J. Wei Stable solutions of the Allen-Cahn equation in dimension 8 and minimal cones, Preprint arXiv:1102.3446v1 (2011).

[33] O. Savin, Regularity of flat level sets in phase transitions, Ann. of Math. (2) 169(2009), no.1, 41-78.

[34] L. Simon, Entire solutions of the minimal surface equation, J. Differential Geometry 30 (1989), 643-688.

[35] J. Simons, Minimal varieties in riemannian manifolds, Ann. of Math. (2) 88(1968), $62-105$.

[36] X.-J. Wang, Convex solutions to the mean curvature flow., Preprint arXiv.org/0404326. To appear in Ann. of Math. 
Manuel del Pino

Departamento de Ingeniería Matemática and Centro de Modelamiento Matemático (UMI 2807 CNRS)

Universidad de Chile

Casilla 170 Correo 3

Santiago

Chile.

e-mail: delpino@dim.uchile.cl

Juncheng Wei

Department of Mathematics

Chinese University of Hong Kong

Shatin

Hong Kong

e-mail: wei@math.cuhk.edu.hk

Received: May 28, 2011. 